\title{
Urinary clusterin and cystatin B as biomarkers of tubular injury in dogs following envenomation by the European adder
}

Gordin, E.

2021-01

Gordin , E , Gordin , D , Viitanen , S , Szlosek, D , Coyne , M , Farace , G, Murphy , R , Quinn , J , Yerramilli , M , Yerramilli , M \& Spillmann , T 2021 , ' Urinary clusterin and cystatin $B$ as biomarkers of tubular injury in dogs following envenomation by the European adder ' , Research in Veterinary Science, vol. 134 , pp. 12-18 . https://doi.org/10.1016/j.rvsc.2020.11.019

http://hdl.handle.net/10138/337951

https://doi.org/10.1016/j.rvsc.2020.11.019

cc_by_nc_nd

acceptedVersion

Downloaded from Helda, University of Helsinki institutional repository.

This is an electronic reprint of the original article.

This reprint may differ from the original in pagination and typographic detail.

Please cite the original version. 
Original Article

Urinary Clusterin and Cystatin B as biomarkers of tubular injury in dogs following envenomation by the European adder

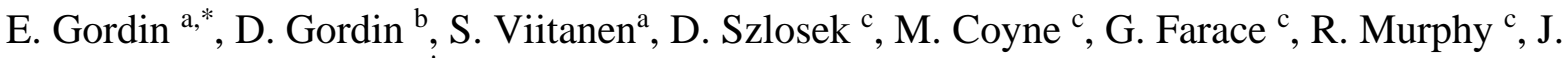
Quinn ${ }^{\mathrm{c}}$, Ma. Yerramilli ${ }^{\mathrm{c} \dagger}, \mathrm{Mu}$. Yerramilli ${ }^{\mathrm{c}}, \mathrm{T}$. Spillmann ${ }^{\mathrm{a}}$

${ }^{a}$ Faculty of Veterinary Medicine, Department of Equine and Small Animal Medicine, Internal Medicine Section, University of Helsinki, P.O. Box 57, 00014 University of Helsinki, Finland

${ }^{b}$ Abdominal Center Nephrology, Helsinki University Hospital, Folkhälsan Research Center, University of Helsinki, Helsinki, Finland

${ }^{c}$ IDEXX Laboratories, Inc., One IDEXX Drive, Westbrook, Maine, 04092, United States of America

† Dr Yerramilli's current address is: Novartis Institutes for BioMedical Research, Inc., 250 Massachusetts Avenue, Cambridge, MA, 02139, United States of America

- Corresponding author. Tel.: +3585 $\underline{04489978}$

E-mail address: emilia.gordin@ helsinki.fi 


\begin{abstract}
Diagnosing acute kidney injury remains a challenge since the established renal biomarkers, serum creatinine $(\mathrm{sCr})$ and symmetric dimethylarginine (SDMA) reflect glomerular function and not tubular injury. Sensitive tubular markers such as urinary clusterin (uClust) and cystatin B (uCysB) have been proposed to detect AKI at an earlier stage. Since envenomation by the European adder (Vipera berus berus) could serve as a spontaneous disease model of AKI we investigated these new biomarkers in affected dogs. Concentrations of uClust and $\mathrm{uCysB}$ as well as $\mathrm{sCr}$ and SDMA were analyzed retrospectively in stored samples from 26 dogs with snake envenomation and 13 healthy controls. Higher concentrations of uClust $(P<0.012)$ and uCysB $(P<0.001)$ were observed in the snakeenvenomed group. Normalization of uClust and $\mathrm{uCysB}$ to urinary creatinine did not alter the results. No differences were observed in $\mathrm{sCr}$ and SDMA between the snake-envenomed group and the healthy control group. Spearman rank correlation analysis revealed a strong association of uClust with uCysB in the snake-envenomed dogs $(r=0.75 P<0.001)$ but not in the healthy controls. The high percentage of snake-envenomed dogs with increased uClust and $\mathrm{uCysB}$ concentrations in the absence of increased sCr and SDMA suggests renal tubular injury in the affected dogs. Larger prospective case-controlled studies are warranted to evaluate the clinical utility and prognostic value of these biomarkers.
\end{abstract}

Keywords: Acute kidney injury; Canine; Envenomation; European adder; Tubular biomarker.

\title{
Introduction
}


Diagnosing acute kidney injury remains a challenge since the standard biomarkers, urea and creatinine, reflect kidney function and not tubular injury. Loss of kidney function, measured as a decrease in glomerular filtration rate (GFR), is not detectable immediately after AKI with surrogate markers of GFR (Endre et al., 2011). Subclinical kidney injury can also occur without reduction of GFR (Vanmassenhove et al., 2019). Further, sCr is an insensitive marker of GFR alterations and does not become increased above reference until a large fraction of kidney function already is lost (Finco 1997).

The lack of early and robust diagnostic tests for AKI has resulted in an intense search of new biomarkers to enable earlier diagnosis and earlier intervention to prevent further injury (Hokamp et al., 2016; Parikh et al., 2017). Two novel AKI biomarkers, urinary clusterin (uClust) and urinary cystatin B (uCysB) have been shown to be sensitive for detection of gentamicin-induced renal proximal tubular toxicity (Yerramilli et al., 2016). Further, dogs with AKI caused by leishmaniasis or hemorrhagic shock treated with colloids showed increased uClust ( Boyd et al., 2019; Garcia-Martinez et al., 2012). Clusterin is a 76$80 \mathrm{kDa}$ protein that is expressed in numerous other tissues in addition to renal tissue. The glycosylation pattern is different between kidney specific clusterin and the plasma isoform, making it possible to measure kidney specific uClust (Yerramilli et al., 2016). Cystatin B has previously been purified from supernatant collected from ruptured canine kidney cells but not from stressed kidney cells (Yerramilli et al., 2016). The same authors report, that cystatin B found in the serum or urine originates from ruptured kidney cells, which in active kidney injury most likely is due to apoptosis and necrosis of epithelial cells in the proximal tubule.

The venom of the European adder (Vipera berus berus) consists of a mixture of approximately 25 different proteins and peptides with hemotoxic activity and to a lesser extent neuro-, myo-, and cytotoxic properties (Bocian et al., 2016). The envenomation may cause a number of clinical and biochemical alterations including changes in renal parameters 
in a subset of affected dogs. The Veterinary Poisons Information Center in the UK reported 422 dogs with envenomation by $V$. berus berus. Of the envenomed dogs, $3.4 \%$ showed renal pathology (increased creatinine, urea, hematuria or renal failure) and the mortality rate was 4.6\% (Sutton et al., 2011). On the other hand, in a Swedish cohort comprising 53 dogs, none of the affected patients died nor had increases in sCr values above reference intervals (Lervik et al., 2010). The effect of adder venom on canine kidneys has been investigated in more detail in two studies that suggested envenomation by the European adders have nephrotoxic properties in dogs (Palviainen et al., 2012; Palviainen et al., 2013). Urinary alpha-1antitrypsin and beta-2-microglobulin concentrations were higher in the urine of envenomed dogs in comparison to healthy controls suggesting tubular injury (Palviainen et al., 2012). Also the urinary ratios of $\gamma$-glutamyl transpeptidase/creatinine (uGgt/ $\mathrm{uCr}$ ), alkaline phosphatase (uAlp/ uCr), and protein/creatinine (UPC) were significantly higher in envenomed dogs, indicating renal injury (Palviainen et al., 2013). However, uGgt/ uCr and uAlp/ uCr ratios did not differ between AKI and chronic kidney disease in another study (Nivy et al., 2017).

Two previous studies in dogs envenomed by adders (Vipera berus berus and Vipera palestinae) reported that clinical signs were more severe in dogs with bites on limbs compared to those with bites on other parts of the body (Segev et al., 2004; Vestberg et al., 2017). However, the effect of location and the time between sample collection and snake bite on kidney biomarkers have not been studied in dogs bitten by Vipera berus berus.

When treating dogs envenomed by adders, colloids have been used to stabilize the hemodynamics in critically ill patients. However, recent studies have raised concern about colloids causing AKI (Boyd et al., 2019; Hayes et al., 2016; Moeller et al., 2016).

The primary objective of this study was to investigate whether dogs bitten by the European adder show increased urinary concentrations of two novel tubular markers, uClust 
and $\mathrm{uCysB}$ as a possible sign of AKI in dogs. The secondary objectives were to investigate whether these novel tubular biomarkers correlate with established biomarkers of renal glomerular function (sCr and SDMA).

\section{Materials and methods}

\section{Study population}

In this descriptive retrospective cohort study, stored serum and urine samples were used from a previous study that included 31 client-owned dogs with a high suspicion of envenomation by $V$. berus berus and treated at the University of Helsinki Veterinary Teaching Hospital during 2009 (Palviainen et al., 2013). The suspicion of a snake bite was based either on the owner observing the adder bite or observing an adder close to the dog and typical signs such as swelling and pain of the bitten area. Dogs with a known history of renal disease were excluded. Four dogs in the snake- envenomed group were excluded from this study due to an unlikely snake bite based on thorough evaluation of all medical records $(\mathrm{n}=$ 4), one had a history of previous azotemia $(n=1)$ and in one case there was insufficient sample volume $(n=1)$. Thus, serum and urine samples of 25 dogs with confirmed envenomation remained in the study.

In 2019, serum and urine samples were collected from an additional 13 dogs who were identified as healthy based on physical examination, complete blood count, blood chemistry profile and urinalysis. Sampling of healthy dogs was approved by the Animal Experiment Board in Finland (ESAVI/3381/04.01.07/2017). All owners of the participants gave their written informed consent.

Sample handling

Blood and urine samples were collected from snake-envenomed dogs during hospitalization. Time from envenomation to sample collection and location of snake bite 
were recorded as well as administration of colloids. Serum was separated after centrifugation (1300 g, $10 \mathrm{~min}$, and room temperature). The serum concentration of $\mathrm{sCr}$ in envenomed dogs was originally measured using a clinical chemistry analyzer (Konelab 30i, Thermo Fisher). For this study, $\mathrm{sCr}$ concentrations from envenomed dogs were re-measured after storage by a colorimetric method, Jaffe's reaction using picrate at alkaline pH (AU680 Chemistry Analyzer, Beckman Coulter, Inc.) and compared with previous test results to assess the influence of storage on the results of the new analysis. There was a high correlation for $\mathrm{sCr}$ analyzed in Helsinki (2009) and at IDEXX Laboratories (2019) (Spearman's $\rho=0.87, \mathrm{P}$ $<0.001)$. Serum from healthy control dogs was analyzed using a clinical chemistry analyzer (AU680 Chemistry Analyzer, Beckman Coulter, Inc.). SDMA was determined using a validated, commercially available, high-throughput immunoassay (IDEXX SDMA® Test, IDEXX Laboratories, Inc.).

Voided, free-catch urine samples were collected from envenomed dogs while urine samples from healthy controls were either voided or collected by ultrasound guided cystocentesis. Urinary creatinine and protein concentrations were measured using routine laboratory methods. All serum and urine samples were stored in $-80{ }^{\circ} \mathrm{C}$ until analyses. Concentrations of uClust and $\mathrm{uCysB}$ were measured using sandwich format immunoassays as previously described by Yerramilli et al (Yerramilli et al., 2016).

\section{Statistical analysis}

All data were calculated using an open source available software $(\mathrm{R}$ version 3.6.1. for Windows). Spearman rank correlation was performed to compare associations for all biomarkers against each other. Significance testing for the Spearman rank correlation was done to test for a slope of zero. The Wilcoxon rank sum test was used to compare the distributions of the continuous variables between the snake-envenomed and healthy control populations. When normalization by urinary creatinine was used, it was noted. Ordinary least 
squares regression was performed to understand the changes in the AKI markers according to location and time of the snakebite. Residuals were graphically analysed to assess uniformity, and transformation was not necessary. Statistical significance was defined as $P<0.05$.

The acute kidney injury markers uClust and uCysB were analyzed. In this study population, 10/13 (76.9\%) of the healthy controls and 10/25 (40.0\%) of the snakebite sample had uClust values below the limit of quantification (LOQ $=70 \mathrm{ng} / \mathrm{mL}$ ) for the assay. For uCysB, 9/13 (69.2\%) of healthy controls and 5/25 (20.0\%) of snake-envenomed dogs had values below the limit of quantification for the assay ( $\mathrm{LOQ}=45 \mathrm{ng} / \mathrm{mL}$ ). With such a large number of values below the limit of quantification, advanced imputation methods such as mixture models or multiple imputation led to erroneous values far above the limit of quantification (which is bounded by 0 and the LOQ for the assay). For this reason and to be conservative in our comparisons, the values below the LOQ were imputed using the LOQ value (70 ng/mL for uClust and $45 \mathrm{ng} / \mathrm{mL}$ for $\mathrm{uCysB}$ ).

\section{Results}

Study population

Twenty-five dogs with snake envenomation and 13 healthy controls were included in the study. Differences in the age distribution were found between the snake-envenomed group (median: 3.8 years, IQR: 1.9 - 7.4) and healthy controls (median: 6.7 years, IQR: 3.8 9.9, Wilcoxon Rank Sum Test $\mathrm{P}<0.023$; Table 1). No differences were observed in the distribution of the weights between the snake-envenomed group (median: $19.0 \mathrm{~kg}$, IQR: $9.4-$ 25.7) and healthy control (median: $22.5 \mathrm{~kg}$, IQR: $8.7-29.1$, Wilcoxon Rank Sum Test $\mathrm{P}=$ 0.451; Table 1).

Routine serum renal markers and novel urinary renal biomarkers 
When comparing the biomarkers between the snake-envenomed and healthy dogs, uClust was higher in the snake-envenomed dogs, regardless of normalization by $\mathrm{uCr}$ (Wilcoxon Rank Sum Test $P<0.012$; Table 1; Fig. 1). Similarly, uCysB was higher in the snake-envenomed group than the healthy control group, whether or not urinary normalization factors were used (Wilcoxon Rank Sum Test $P<0.001$; Table 1; Fig. 2). No differences in the renal biomarkers $\mathrm{sCr}(P=0.948)$ and SDMA $(P=0.994)$ between the two groups were observed, respectively (Table 1; Fig.3). Urine protein: creatinine was higher in the snakeenvenomed group $(P=0.001$; Table 1$)$.

An association was found between UPC and both urinary AKI markers with and without normalization for uCr (Spearman's $\rho_{u C l u s t}=0.511, P<0.015$, Spearman's $\rho_{u C y s B}=$ $0.671, P<0.001$; Table 2 ). In envenomed dogs $\mathrm{sCr}$ was positively correlated to uClust, but this finding was not observed when normalizing uClust with uCr. A negative correlation was found between $\mathrm{sCr}$ and $\mathrm{uClust} / \mathrm{uCr}$ in healthy dogs, not observed when not normalizing uClust to uCr. Neither of the renal biomarkers sCr and SDMA were correlated with uCyst B regardless of normalization for $\mathrm{uCr}$ in either the healthy control or snake-envenomed group (Table 2).

Location of snakebite was evaluated by descriptive statistics with most of the bites occurring on the head $(n=16)$ and limbs $(n=8)$ and the least on the neck $(n=1)$. When comparing serum and urinary biomarker levels between the regions of the snakebite (head, limb), no differences in the distributions between head and limbs were observed for $\mathrm{sCr}$, SDMA or uClust (Table 3). However, differences were observed between uCysB levels in dogs with snake bites in the head and limb (Wilcoxon Rank Sum Test $\mathrm{P}<0.012$; Table 3).

Time from envenomation was weakly correlated with uClust (Spearman's $\rho=$ 0.521, $P<0.009)$. In contrast, uCysB was not correlated with time from envenomation (Spearman's $\rho=0.241, P=0.256$; Table 4). 
A total of $28.0 \%(7 / 25)$ of the snake-envenomed dogs received colloidal treatment with $4 \%$ succinylated gelatin (gelo, Gelofusine B. Braun Melsungen AG). Higher concentrations in both uClust and $\mathrm{uCysB}$ were observed within the gelo group compared to the non- colloidal group (Wilcoxon Rank Sum Test $P<0.005$; Figs. 4A, B). There was no significant difference in the time from envenomation to sample collection between the gelo group (median: 1.90 days, IQR: $1.14-2.75)$ and the non- colloidal group (median: 0.92 days, IQR: $0.84-2.00$, Wilcoxon Rank Sum $P=0.174)$. Neither were there differences in clinical outcome nor in sCr and SDMA in the gelo and the non- colloidal groups. (Figs. $4 \mathrm{C}, \mathrm{D}$ ).

\section{Discussion}

The novel renal tubular AKI biomarkers uClust and uCysB were significantly higher in the group of dogs bitten by the European adder compared to healthy control dogs. Further, uClust and uCysB were strongly correlated to each other in the snake-envenomed group but not in the control group. These findings suggest renal tubular injury in dogs envenomed by European adders. Concentrations of $\mathrm{sCr}$ and SDMA, both surrogate markers of GFR, were not observed to be different between the two groups nor to be correlated to uClust or uCysB. Thus, envenomation by the common European adder results primarily in subclinical AKI, but not necessarily in decreased renal function measured as increases in $\mathrm{sCr}$ and SDMA. In human medicine, elevations of tubular injury biomarkers in the absence of changes in traditional biomarkers of renal function (sCrea and UO), were shown to have an association with adverse outcomes (Haase et al., 2011; Joannidis et al., 2019; Nickolas et al., 2012). Recent studies also revealed worse outcomes in AKI patients being either under- or especially over hydrated (Bagshaw et al., 2008; Mehta et al., 2016; Salahuddin et al., 2017). Whether biomarkers of AKI could be used to support treatment decisions e.g. against the use of potentially nephrotoxic agents such as NSAIDs and colloids in dogs, still needs to be 
studied. Also, the possible support of AKI biomarkers for evaluating intravenous fluid therapy requires further investigation, as it has been routinely used as a part of the supportive therapy for eg. snake envenomed dogs.

The region of the snake bite did not play a major role in changes in AKI biomarkers in our study. Interestingly, two previous studies reported more severe clinical signs in dogs bitten on the limbs compared to other areas of the body. In the first study, $8 / 17$ dogs bitten by $V$. berus berus, developed cardiac arrhythmias. Four of these dogs had bites on the limbs and all four became arrhythmic (Vestberg et al., 2017). In the second study, dogs bitten by Vipera palasteniae, a close relative to Vipera berus berus, showed an increased mortality rate in dogs bitten on limbs compared to other areas of the body (Segev et al., 2004). The authors suggest that it might be possible that limb envenomation enables a wider and more rapid toxin distribution throughout the body compared to bites on for example the head, where more soft tissue is capable of absorbing the venom. In our study, we found that only uCysB but not uClust, $\mathrm{sCr}$ or SDMA concentrations were higher in dogs bitten in the limbs compared to bites on the head. One reason for the lack of an association between bite site and elevation in uClust could be explained by either a possibly slower increase of this biomarker compared to $\mathrm{uCysB}$ or the study being too underpowered to draw conclusion on this aspect. Since in most cases the samples were collected soon after the bite, future studies should focus on repeated sampling for a longer period to investigate the aspect of different timely elevations of urinary AKI biomarkers.

In this study, time from snake envenomation to sample collection varied between 2.5 $\mathrm{h}$ and 4 days but did not have a significant effect on uClust or uCysB levels. There are currently no published data regarding the volume of distribution and half-life of the toxic components of $V$. berus berus toxin in dogs. It can be speculated that the lack of decrease in uClust and $\mathrm{uCysB}$ with increased time from envenomation could be due to the toxin having a 
long half-life, exerting its toxic effects for a prolonged period of time. It might also reflect an ongoing active process initiated by the envenomation. The half-lives of uClust and uCysB have not been studied in dogs so far, and long biomarker half-lives could also explain our finding. Further studies are needed to understand this better.

More than one fourth (28\%) of the snake-envenomed dogs were treated with gelo infusion in addition to crystalloids. The AKI markers were statistically significantly higher in these patients in comparison to non-colloid treated dogs. There were no differences in clinical outcome between the gelo and the non- colloidal groups. A previous study reported an effect of colloids on renal health and gelo administration was shown to increase uClust levels in a set of dogs with hemorrhage induced shock (Boyd et al., 2019). However, due to the retrospective nature of this study, the indication for colloid treatment was not known. The differences in the gelo group could be due to the treatment or that the patients were at a more critical condition leading to the use of gelo in addition to crystalloid infusion. These findings have anyhow no effect on the interpretation of the urinary biomarker results and can therefore be regarded as minor limitations.

The UPC was higher in the snake-envenomed group compared to the group of healthy controls. Previous studies in humans and dogs with snake bites have shown similar findings (Hrovat et al., 2013; Kanjanabuch et al., 2008; Palviainen et al., 2012). Proteins in the urine can be filtered plasma proteins or originate from the kidneys and the lower urinary tract. Primary glomerular dysfunction results in abnormal leakage of high and intermediate molecular weight proteins into the urine. Small and to some extent intermediate molecular weight proteins are however freely filtered thru intact glomerular filtration barriers and are absorbed by the proximal tubules in health. Tubular damage results in inability to reabsorb low molecular weight proteins and leads to leakage of proteins from injured epithelial cells into urine (D'Amico et al., 2003). Measurement of UPC cannot be used to determine the type 
or origin of proteins (De Loor et al., 2013). Thus, based on our data, we cannot exclude concurrent glomerular dysfunction in addition to tubular damage, albeit this seems unlikely given the observed low magnitude proteinuria (Littman et al., 2013).

There are a number of limitations in our study. The samples from both groups were stored at $-80 \mathrm{C}^{\circ}$ but samples from envenomed dogs were stored for 10 years. This could have influenced the results. However, the values of the investigated biomarkers are on average higher in the archived samples than in the samples from control dogs reducing the likelihood that the storage time was of influence on the results. The healthy controls consisted of randomly selected dogs owned by staff members of the University of Helsinki Veterinary Teaching Hospital. They were older than the snake-envenomed dogs. It is unlikely that the age difference between the two groups affected the outcome of this study, since the risk of having an underlying chronic kidney disease is greater in older dogs ( $\mathrm{O}^{\prime} \mathrm{Neill}$ et al., 2013). Only single point samples were used in this study. The sensitivity of sCr in detecting AKI is higher with serial monitoring and using cut off values of increases in sCr of 26.4 $\mu \mathrm{mol} / \mathrm{L}(0.3 \mathrm{mg} / \mathrm{dL})$ as proposed by the International Renal Interest Society's guidelines (International Renal Interest Society 2016). Future studies should include serial monitoring of sCr that might reveal possible functional decline in dogs envenomed by the European adder. Lastly, between $20 \%$ and $77 \%$ of samples had values that were below the limit of quantification in the envenomed and control groups for uClust and $\mathrm{uCysB}$, respectively. Because of this, imputation and rank-based procedures were used to handle the data. With such high levels of imputation used, there is a risk of not knowing the true distribution of the data. To mitigate this risk, a conservative form of imputation was applied using the limit of quantification (LOQ).

\section{Conclusion}


Our results show increased uClust and uCysB concentrations in dogs with snakebite envenomation by the European adder, suggesting renal tubular injury in the affected dogs. No increases were observed in the established markers of renal glomerular function, $\mathrm{sCr}$ and SDMA, highlighting the need for future studies on renal biomarkers in dogs.

\section{Conflict of interest}

These authors have an affiliation to the commercial funders of this research, as employees of IDEXX Laboratories, Inc. The work presented in this study was supported in part by IDEXX Laboratories, Inc. Westbrook, ME, USA (https://www.idexx.com/en/about-idexx/). IDEXX Laboratories, Inc. holds certain patent rights relating to assays for the detection of SDMA, clusterin and cystatin-B and manufactures the IDEXX SDMA® Test utilized in this study.

M. Coyne, G. Farace, R. Murphy, J. Quinn, D. Szlosek, M. Yerramilli, and M. Yerramilli were employed by IDEXX Laboratories, Inc., during the time of the study.

\section{Acknowledgements}

Preliminary results were presented as an Abstract at the $25^{\text {th }}$ International Emergency \& Critical Care Symposium, Washington DC, 6-10 September 2019. This work was supported by Arvid and Greta Olin's Fund, Finnish Foundation of Veterinary Research, Finnish Veterinary Research Foundation, Orion Research Foundation and University of Helsinki Doctoral Program in Clinical Veterinary Medicine.

Bagshaw, S.M., Brophy, P.D., Cruz, D., Ronco, C., 2008. Fluid balance as a biomarker: impact of fluid overload on outcome in critically ill patients with acute kidney injury. Critical care 12: 1-3. 
Bocian, A., Urbanik M., Hus K., Lyskowski A., Petrilla V., Andrejcakova Z., Petrillova M., Legath J., 2016. Proteome and Peptidome of Vipera berus berus Venom. Molecules 21: 1398

Boyd, C.J., Claus, M.A., Raisis, A.L., Cianciolo, R., Bosio, E., Hosgood, G., Nabity, M., Mori, T., Barden, A., Sharp, C.R., Smart, L., 2019. Evaluation of biomarkers of kidney injury following 4\% succinylated gelatin and 6\% hydroxyethyl starch 130/0.4 administration in a canine hemorrhagic shock model. Journal of Veterinary Emergency \& Critical Care 29: 132- 142.

D'Amico, G., Bazzi, C., 2003. Pathophysiology of proteinuria. Kidney International 63: 809825.

De Loor, J., Daminet, S., Smets, P., Maddens, B., Meyer, E., 2013. Urinary biomarkers for acute kidney injury in dogs. Journal of Veterinary Internal Medicine 27: 998-1010.

Endre, Z. H., Pickering, J.W., Walker, R.J., 2011. Clearance and beyond: the complementary roles of GFR measurement and injury biomarkers in acute kidney injury (AKI). American Journal of Physiology - Renal Physiology 301: F697-707.

Finco, D. R., 1997. Kidney function. In: Clinical biochemistry of domestic animals, Fifth Edn. Elsevier, USA, pp 441- 484.

Garcia-Martinez, J.D., Tvarijonaviciute, A., Ceron, J.J., Caldin, M., Martinez-Subiela, S., 2012. Urinary clusterin as a renal marker in dogs. Journal of Veterinary Diagnostic Investigation 24: 301-306.

Haase, M., Devarajan, P., Haase-Fielitz, A., Bellomo, R., Cruz, D.N., Wagener, G., Krawczeski, C.D., Koyner, J.L., Murray, P., Zappitelli, M., 2011. The outcome of neutrophil gelatinase-associated lipocalin-positive subclinical acute kidney injury: a multicenter pooled analysis of prospective studies. Journal of the American College of Cardiology 57: 1752-61.

Hayes, G., Benedicenti, L., Mathews, K., 2016. Retrospective cohort study on the incidence of acute kidney injury and death following hydroxyethyl starch (HES 10\% 250/0.5/5: 1) administration in dogs (2007-2010). Journal of Veterinary Emergency \& Critical Care 26: 35-40.

Hokamp, J.A., Nabity, M.B., 2016. Renal biomarkers in domestic species. Veterinary Clinical Pathology 45: 28-56.

Hrovat, A., Schoeman, J.P., de Laat, B., Meyer, E., Smets, P., Goddard, A., Nagel, S., Daminet, S., 2013. Evaluation of snake envenomation-induced renal dysfunction in dogs using early urinary biomarkers of nephrotoxicity. The Veterinary Journal 198: 239-244.

International Renal Interest Society, 2016. Grading of acute kidney injury. http://iriskidney.com/pdf/4_ldc-revised-grading-of-acute-kidney-injury.pdf (accessed 12 May 2020).

Joannidis, M., Forni, L.G., Haase, M., Koyner, J., Shi, J., Kashani, K., Chawla, L.S., Kellum J.A., 2019. Use of cell cycle arrest biomarkers in conjunction with classical markers of acute kidney injury. Critical care medicine 47: e820.

Kanjanabuch, T., Sitprija, V., 2008. Snakebite nephrotoxicity in Asia. Seminars in Nephrology 28: 363-372.

Lervik, J.B., Lilliehook, I., Frendin, J.H., 2010. Clinical and biochemical changes in 53 Swedish dogs bitten by the European adder--Vipera berus. Acta Veterinaria Scandinavica 52: 26.

Littman, M.P., Daminet, S., Grauer, G.F., Lees, G.E., van Dongen, A.M., 2013. Consensus recommendations for the diagnostic investigation of dogs with suspected glomerular disease. Journal of Veterinary Internal Medicine 27 Suppl 1: S19-26. 
Mehta, R. L., Burdmann, E.A., Cerdá, J., Feehally, J., Finkelstein, F., García-García, G., Godin, M., Jha, V., Lameire, N.H., Levin N.W., 2016. Recognition and management of acute kidney injury in the International Society of Nephrology 0by25 Global Snapshot: a multinational cross-sectional study. The Lancet 387: 2017-2025.

Moeller, C., Fleischmann, C., Thomas-Rueddel, D., Vlasakov, V., Rochwerg, B., Theurer, P., Gattinoni, L., Reinhart, K., Hartog, C.S., 2016. How safe is gelatin? A systematic review and meta-analysis of gelatin-containing plasma expanders vs crystalloids and albumin. Journal of Critical Care 35: 75-83.

Nickolas, T.L., Schmidt-Ott, K.M., Canetta, P., Forster, C., Singer, E., Sise, M., Elger, A., Maarouf,O., Sola-Del Valle, D.A., O'Rourke, M., 2012. Diagnostic and prognostic stratification in the emergency department using urinary biomarkers of nephron damage: a multicenter prospective cohort study. Journal of the American College of Cardiology 59: 246-255.

Nivy, R., Avital, Y., Aroch, I., Segev, G., 2017. Utility of urinary alkaline phosphatase and gamma- glutamyl transpeptidase in diagnosing acute kidney injury in dogs. The Veterinary Journal 220: 43-47.

O'Neill, D.G., Elliott, J., Church, D.B., McGreevy, P.D., Thomson, P.C., Brodbelt, D.C., 2013. Chronic kidney disease in dogs in UK veterinary practices: prevalence, risk factors, and survival. Journal of Veterinary Internal Medicine 27: 814-821.

Palviainen, M., Raekallio, M., Vainionpaa, M., Kosonen, S., Vainio, O., 2012. Proteomic profiling of dog urine after European adder (Vipera berus berus) envenomation by two-dimensional difference gel electrophoresis. Toxicon 60: 1228- 1234.

Palviainen, M., Raekallio, M., Vainionpaa, M., Lahtinen, H., Vainio, O., 2013. Evaluation of renal impairment in dogs after envenomation by the common European adder (Vipera berus berus). The Veterinary Journal 198: 723-724.

Parikh, C.R., Mansour, S.G., 2017. Perspective on Clinical Application of Biomarkers in AKI. Journal of the American Society of Nephrology 28: 1677-1685.

Salahuddin, N., Sammani, M., Hamdan, A., Joseph, M., Al-Nemary, Y., Alquaiz, R., Dahli, R., Maghrabi, K., 2017. Fluid overload is an independent risk factor for acute kidney injury in critically Ill patients: results of a cohort study. BMC nephrology 18: 45.

Segev, G., Shipov, A., Klement, E., Harrus, S., Kass, P., Aroch, I., 2004. Vipera palaestinae envenomation in 327 dogs: a retrospective cohort study and analysis of risk factors for mortality. Toxicon 43: 691-699.

Sutton, N.M., Bates, N., Campbell, A., 2011. Canine adder bites in the UK: a retrospective study of cases reported to the Veterinary Poisons Information Service. Veterinary Record 169: 607.

Vanmassenhove, J., Van Biesen, W., Vanholder, R., Lameire, N., 2019. Subclinical AKI: ready for primetime in clinical practice?. Journal of Nephrology 32: 9-16.

Vestberg, A.R., Tidholm, A., Ljungvall, I., 2017. Twenty-four-hour ambulatory electrocardiography characterization of heart rhythm in Vipera berus-envenomed dogs. Acta Veterinaria Scandinavica 59: 28.

Yerramilli, M., Farace, G., Quinn J., Yerramilli, M., 2016. Kidney Disease and the Nexus of Chronic Kidney Disease and Acute Kidney Injury: The Role of Novel Biomarkers as Early and Accurate Diagnostics. Veterinary Clinics of North America: Small Animal Practice 46: 961-993. 
Table 1

Routine serum and urinary parameters and novel renal biomarkers in 25 snake-envenomed dogs and 13 healthy control dogs.

\begin{tabular}{|c|c|c|c|c|c|}
\hline \multirow[b]{2}{*}{ Category } & \multicolumn{2}{|c|}{$\begin{array}{l}\text { Snake-envenomed } \\
6 \mathrm{~m}, 5 \mathrm{mn}, 10 \mathrm{f}, 4 \mathrm{fn}\end{array}$} & \multicolumn{2}{|c|}{$\begin{array}{l}\text { Healthy controls } \\
2 \mathrm{~m}, 2 \mathrm{mn}, 3 \mathrm{f}, 6 \mathrm{fn}\end{array}$} & \multirow[b]{2}{*}{$P^{\mathrm{a}}$} \\
\hline & Median & IQR & Median & IQR & \\
\hline Age (years) & 3.8 & $1.9-7.4$ & 6.7 & $3.8-9.9$ & 0.023 \\
\hline $\begin{array}{l}\text { Bodyweight } \\
(\mathrm{kg})\end{array}$ & 19.0 & $9.4-25.7$ & 22.5 & $8.7-29.1$ & 0.451 \\
\hline $\begin{array}{l}\mathrm{sCr}( \\
\mu \mathrm{mol} / \mathrm{L})\end{array}$ & 61.6 & $44.0-70.4$ & 70.4 & $61.6-88.0$ & 0.948 \\
\hline $\begin{array}{l}\text { SDMA } \\
(\mu \mathrm{mol} / \mathrm{L})\end{array}$ & 2.9 & $2.2-3.6$ & 3.6 & $3.3-4.4$ & 0.994 \\
\hline $\begin{array}{l}\text { uClust } \\
\text { (ng/mL) }\end{array}$ & 199.0 & $\begin{array}{l}70.0- \\
1187.0\end{array}$ & 70.0 & $70.0-70.0^{b}$ & 0.012 \\
\hline uClust / uCr & 697.3 & $\begin{array}{r}229.6- \\
4337.8\end{array}$ & 71.4 & $32.8-191.0$ & 0.001 \\
\hline $\begin{array}{l}\text { uCysB } \\
\text { (ng/mL) }\end{array}$ & 188.0 & $\begin{array}{r}64.0- \\
443.0\end{array}$ & 45.0 & $45.0-61.0$ & 0.001 \\
\hline $\begin{array}{l}\text { uCysB / } \\
\text { uCr }\end{array}$ & 665.9 & $\begin{array}{r}207.2- \\
1497.8\end{array}$ & 46.7 & $21.1-56.8$ & 0.001 \\
\hline UPC & 0.68 & $0.22-1.77$ & 0.09 & $0.06-0.12$ & 0.001 \\
\hline USG & 1.01 & $1.01-1.02$ & 1.04 & $1.02-1.04$ & 0.999 \\
\hline
\end{tabular}

$\mathrm{m}$, male intact; $\mathrm{mn}$, male neutered; f, female; fn, female neutered, uClust, urinary clusterin; uCysB, urinary cystatin B; sCr, serum creatinine; SDMA, symmetric dimethyl arginine; $\mathrm{uCr}$, urinary creatinine; UPC, urine protein- creatinine ratio; USG, urine specific gravity. ${ }^{\text {a } P}$ based off the Wilcoxon Rank Sum Test

${ }^{\mathrm{b}}$ In control dogs $76.9 \%$ had uClust levels below the limit of detection and imputed at a value of $70 \mathrm{ng} / \mathrm{mL}$. 


\section{Table 2}

Correlation between age, bodyweight, renal biomarkers and UPC of 25 snake-envenomed and 13 healthy dogs

\begin{tabular}{|c|c|c|c|c|c|c|c|c|c|}
\hline \multirow[b]{3}{*}{ Category } & \multirow[b]{3}{*}{ Status } & \multicolumn{8}{|c|}{ Spearman's Correlation Coefficient } \\
\hline & & \multicolumn{2}{|c|}{ uClust (ng/dL) } & \multicolumn{2}{|c|}{ uClust /uCr } & \multicolumn{2}{|c|}{ uCysB (ng/dL) } & \multicolumn{2}{|c|}{ uCysB /uCr } \\
\hline & & Spearman's $\rho$ & $\mathrm{P}$ & $\begin{array}{c}\text { Spearman's } \\
\rho\end{array}$ & $\mathrm{P}$ & $\begin{array}{c}\text { Spearman's } \\
\rho\end{array}$ & $\mathrm{P}$ & $\begin{array}{c}\text { Spearman's } \\
\rho\end{array}$ & $\mathrm{P}$ \\
\hline \multirow[t]{2}{*}{ Age (years) } & Envenomed & -0.11 & 0.609 & -0.369 & 0.077 & -0.092 & 0.670 & -0.408 & 0.049 \\
\hline & Healthy & -0.134 & 0.663 & -0.055 & 0.863 & -0.027 & 0.931 & -0.071 & 0.821 \\
\hline \multirow{2}{*}{$\begin{array}{l}\text { Bodyweight } \\
(\mathrm{kg})\end{array}$} & Envenomed & -0.025 & 0.906 & -0.183 & 0.381 & -0.054 & 0.799 & -0.161 & 0.441 \\
\hline & Healthy & -0.193 & 0.527 & -0.669 & 0.012 & 0.222 & 0.467 & -0.446 & 0.127 \\
\hline \multirow[t]{2}{*}{$\mathrm{sCr}(\mu \mathrm{mol} / \mathrm{L})$} & Envenomed & 0.465 & 0.019 & 0.383 & 0.059 & 0.210 & 0.313 & 0.084 & 0.689 \\
\hline & Healthy & -0.418 & 0.155 & -0.596 & 0.032 & -0.041 & 0.895 & -0.496 & 0.085 \\
\hline \multirow{2}{*}{$\begin{array}{l}\text { SDMA ( } \mu \mathrm{mol} \\
/ \mathrm{L})\end{array}$} & Envenomed & 0.107 & 0.612 & 0.170 & 0.417 & 0.038 & 0.856 & 0.071 & 0.735 \\
\hline & Healthy & -0.361 & 0.225 & -0.034 & 0.913 & -0.103 & 0.739 & 0.227 & 0.457 \\
\hline \multirow{2}{*}{$\begin{array}{l}\text { uClust } \\
\text { (ng/mL) }\end{array}$} & Envenomed & --- & --- & --- & --- & 0.749 & $<0.001$ & 0.598 & 0.002 \\
\hline & Healthy & --- & --- & --- & --- & -0.354 & 0.235 & -0.126 & 0.681 \\
\hline \multirow[t]{2}{*}{ uClust / uCr } & Envenomed & --- & --- & --- & --- & 0.706 & $<0.001$ & 0.850 & $<0.001$ \\
\hline & Healthy & --- & --- & --- & --- & -0.242 & 0.426 & 0.500 & 0.085 \\
\hline \multirow{2}{*}{$\begin{array}{l}\text { uCysB } \\
\text { (ng/mL) }\end{array}$} & Envenomed & 0.749 & $<0.001$ & 0.706 & $<0.001$ & --- & --- & --- & --- \\
\hline & Healthy & -0.354 & 0.235 & -0.242 & 0.426 & --- & --- & --- & --- \\
\hline \multirow[t]{2}{*}{$\mathrm{uCysB} / \mathrm{uCr}$} & Envenomed & 0.598 & 0.002 & 0.850 & $<0.001$ & --- & --- & --- & --- \\
\hline & Healthy & -0.126 & 0.681 & 0.500 & 0.085 & --- & --- & --- & --- \\
\hline \multirow[t]{2}{*}{ UPC } & Envenomed & 0.511 & 0.015 & 0.752 & $<0.001$ & 0.671 & $<0.001$ & 0.844 & $<0.001$ \\
\hline & Healthy & 0.323 & 0.282 & 0.361 & 0.225 & 0.170 & 0.580 & 0.339 & 0.257 \\
\hline & & & & & & & & & \\
\hline & & & & & & & & & \\
\hline & & & & & & & & & \\
\hline
\end{tabular}

uClust, urinary clusterin; uCysB, urinary cystatin B; sCr, serum creatinine; SDMA,

symmetric dimethyl arginine; UPC, urine protein- creatinine ratio.

\section{Table 3}

Snakebite location and renal biomarkers in 25 snake-envenomed dogs

\begin{tabular}{|l|c|c|c|c|c|c|c|}
\hline & \multicolumn{2}{|c|}{ Head $(\mathrm{N}=16)$} & \multicolumn{2}{c|}{$\operatorname{Limb}(\mathrm{N}=8)$} & \multicolumn{2}{c|}{ Neck $(\mathrm{N}=1)$} & \\
\hline \multicolumn{1}{|c|}{ Category } & Median & IQR & Median & IQR & Median & IQR & $P^{\mathrm{a}}$ \\
\hline sCr $(\mu \mathrm{mol} / \mathrm{L})$ & 61.6 & $44.0-70.4$ & 57.2 & $50.6-79.2$ & 35.2 & $35.2-35.2$ & 0.512 \\
\hline $\begin{array}{l}\text { SDMA } \\
(\mu \mathrm{mol} / \mathrm{L})\end{array}$ & 2.9 & $2.5-3.6$ & 2.4 & $1.8-3.7$ & 1.8 & $1.8-1.8$ & 0.830 \\
\hline uClust $(\mathrm{ng} / \mathrm{mL})$ & 70.0 & $\begin{array}{c}70.0- \\
716.0\end{array}$ & 409.0 & $\begin{array}{c}168.8- \\
2013.0\end{array}$ & 2347.0 & $\begin{array}{c}2347.0- \\
2347.0\end{array}$ & 0.072 \\
\hline
\end{tabular}


SCr, serum creatinine; SDMA, symmetric dimethyl arginine; uClust, urinary clusterin; uCysB, urinary cystatin B

\section{Table 4}

Correlations of biomarker values by time from snake bite to sample collection

\begin{tabular}{|c|c|c|c|}
\hline & \multicolumn{3}{|c|}{ Spearman's correlation coefficient $\rho$} \\
\hline Type & Estimate & 95\% Confidence Interval & $P$ \\
\hline uClusterin vs. Time & 0.521 & $0.163-0.768$ & 0.009 \\
\hline uCystatin B vs. Time & 0.241 & $-0.160-0.600$ & 0.139 \\
\hline
\end{tabular}

Note: A Spearman's Correlation Test was conducted to compare distributions of the four biomarkers values between suspected and confirmed snake bite.

\section{Figure legends}


Fig. 1. Comparison of urinary clusterin (uClust) in snake-envenomed $(n=25)$ and healthy control dogs ( $\mathrm{n}=13)$. (A) uClust. (B)uClust normalized by urinary creatinine. Whiskers represent 1.5 times the interquartile range.

Fig. 2. Comparison of urinary cystatin $B(u C y s B)$ in snake-envenomed $(n=25)$ and healthy control dogs (n=13). (A) uCysB. (B) uCysB normalized by urinary creatinine Whiskers represent 1.5 times the interquartile range.

Fig. 3. Comparison of routine renal biomarkers in snake-envenomed $(n=25)$ and healthy control dogs (n=13). (A) Serum creatinine. (B) Symmetric dimethylarginine (SDMA). Whiskers represent 1.5 times the interquartile range.

Fig. 4. Association between colloids and the renal biomarkers. (A) Urinary clusterin. (B) Urinary cystatin B. (C) Serum creatinine. (D) Symmetric dimethylarginine (SDMA). Whiskers represent 1.5 times the interquartile range. 